Sharif University of Technology
Scientia Iranica
SCIENTIA
IRAN Transactions B: Mechanical Engineering
www.scientiairanica.com

\title{
Fluid-structure interaction analysis of airflow in pulmonary alveoli during normal breathing in healthy humans
}

\author{
M. Monjezi and M.S. Saidi* \\ School of Mechanical Engineering, Sharif University of Technology, Tehran, Iran.
}

Received 4 May 2015; received in revised form 26 July 2015; accepted 18 October 2015

\section{KEYWORDS \\ FSI; \\ Alveolar wall; \\ Viscoelastic; \\ Stream line; \\ Stress; \\ Strain.}

\begin{abstract}
In this work, the human lung alveoli are idealized by a three dimensional honeycomb like geometry and a fluid-structure analysis is performed to study the normal breathing mechanics. In contrast to previous works in which the inlet flow rate is predefined, in this model, we have applied a negative pressure on the outside surface of the alveolus which causes air to flow in and out of the alveolus. The integration of the experimental curve of breathing flow rate was used to approximate the shape of the external applied pressure. Our Fluid-Structure Interaction (FSI) model has an advantage over other literature since it addresses both the fluid dynamics and solid mechanics, simultaneously. The flow patterns confirmed that there is no circulation in the terminal alveolus. We have applied three distinct material models - linear isotropic elastic, hyperelastic, and viscoelastic - in order to simulate the mechanical behavior of alveolar wall tissue using ADINA software. The hysteresis behavior of the alveolar tissue was well predicted by a compliance diagram of the viscoelastic model while this behavior is not observed in the linear elastic and hyperelastic model. The stress and strain distribution is also obtained and is found to be non-uniform.

(C) 2016 Sharif University of Technology. All rights reserved.
\end{abstract}

\section{Introduction}

In contrast to the improvement achieved in lung upper airway imaging, success in the $3 \mathrm{D}$ imaging of the alveolar region has been restricted because of its small size and low accessibility. For this reason, most researchers have attempted to present a reliable model which could be used instead of image-based models. Tsuda et al. [1] used a simplified expanding and contracting torus surrounding a central channel. Later, Henry et al. [2] developed an axisymmetric model of an alveolated duct which had nine cells around a central channel. A simplified model consisted of a number of

*. Corresponding author. Tel.: +98 2166165558 ;

Fax: +982166000021

E-mail address: mssaidi@sharif.edu (M.S. Saidi) annular rings around a central channel was presented by Darquenne and Paiva [3]. Harrington et al. [4] performed simulations on a bifurcation model of acinar branching. Haber et al. [5] used a spherical cap and performed an analytical simulation. Various models in the literature have considered rigid wall in their simulations. In the last decade, various efforts have started incorporating alveolar wall motion into their analysis. Haber et al. [6] and Sznitman et al. [7] used a self-similar wall motion with a prescribed sinusoidal function.

Oakes et al. [8] performed an experimental study to find the alveolar mechanics. Similarly, Harding and Robinson [9] used numerical simulation to find fluid patterns in the alveoli. It has been shown that major differences arise in both the flow structure and the particle deposition characteristics when the motion of 
the alveolar wall and alveolar duct are not properly included in the model [10]. Hence, the models of the acinus have to be supplemented with appropriate boundary conditions to mimic breathing.

Berg et al. [11] used particle image velocimetry in a compliant replica model of a terminal air sac and observed the noncirculating air flow pattern in the last two generations of the alveolar region. Recently, Li and Kleinstreuer [12] used a space filling polygon shaped alveolar model and investigated the air flow for different wall motions.

In order to consider the natural relation between tissue motion and airflow in the alveoli, the Fluid Solid Interaction (FSI) is applicable. In order to perform an FSI approach it is necessary to use a suitable model for the mechanical properties of alveolar wall tissue. Gefen et al. [13] analyzed the stress distribution in a 2D model of alveolar tissue using finite element analysis. Dailey and Ghadiali [14] have developed an FSI analysis in a 2D model of alveolar sacs. They have assumed both elastic and Kelvin-Voigt viscoelastic models for alveolar tissue. Naturally, a 2D approach cannot adequately account for the effects of a 3D structural environment, including out-of-plane traction forces from neighboring septa.

In the past few years, there have been a number of successful 3D imaging and reconstructed geometries using micro-Computed Tomography (CT) and radiation-based X-ray Tomographic Microscopy (XTM); these have been used for studies on lung development [15], acinar morphometry [16,17], and acinar mechanics $[18,19]$.

However, these images need some imageprocessing techniques to reconstruct the real 3D geometry, and these are usually complex and time-consuming. So, using an idealized geometry allows us to avoid interpreting complex real geometries and to easily simulate different conditions.

In this paper an FSI model is used to study normal breathing airflow in a 3D honeycomb like geometry of alveoli. For the mechanical properties of alveolar tissue we have used more realistic models according to experimental data previously reported. Different material models of elastic, nonlinear elastic, and viscoelastic are compared with each other. Viscoelasticity is considered by quasi-linear theory and the application of a reduced relaxation function.

The innovative aspects of this research include the development of a computational model that considers the transient flow fields generated by airway wall deformation and fully coupled fluid-structure interactions at the interface on a 3D model of alveolus (with a physiologically-based geometry), more physiological boundary conditions in contrast to sinusoidal pressure load applied by Dailey and Ghadiali [14] and adding the alveolar surface liquid lining and surfactant by including their effect in the material model.

This model provides a possible tool to predict the effect of mechanical properties of the alveolar wall on the distribution of stress and strain.

The remainder of the article is organized in the following sections. Section 2 provides the numerical methodology including the geometry, governing equations, material properties, and boundary conditions. Section 3 presents the results of our simulations and Section 4 summarizes the results in a conclusion.

\section{Methodology}

\subsection{Geometry}

The Scanning Electron Micrographic (SEM) images confirm that alveolus displays a hexagonal-like morphology with thin walls [20]. Based on this observation, our alveolus is a 3D hexagonal honeycomb like geometry which is more accurate than 2D models. Our geometry is similar to Kumar ones [21] which consists of 18 regular hexahedra connected together with a single entrance to represent terminal alveoli. A schematic of this 3D geometry is shown in Figure 1. Since there are no sharp corners in the alveolar morphology, the alveolar corners are rounded to avoid computational artifact. If we cut this geometry by two certain symmetry planes, one-sixth of this geometry is selfsimilar. So, we have used this kind of symmetry to reduce the computational time in our simulation (Figure 2). The length of each side of the hexagon is $80 \mu \mathrm{m}$ and the total length of the alveolar sac is 1000 $\mu \mathrm{m}$.

To represent normal alveolar wall tissue, we have considered an $8 \mu \mathrm{m}$ thickness wall according to [13]. It is noteworthy that in some interstitial diseases the alveolar wall thickness may be significantly larger than $8 \mu \mathrm{m}$.

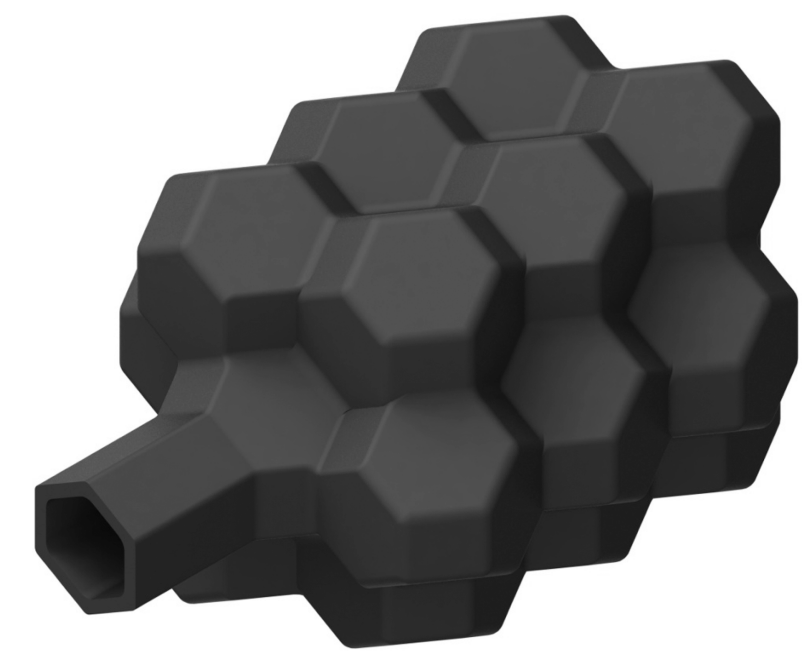

Figure 1. 3D alveolar sac geometry with a single entrance. 


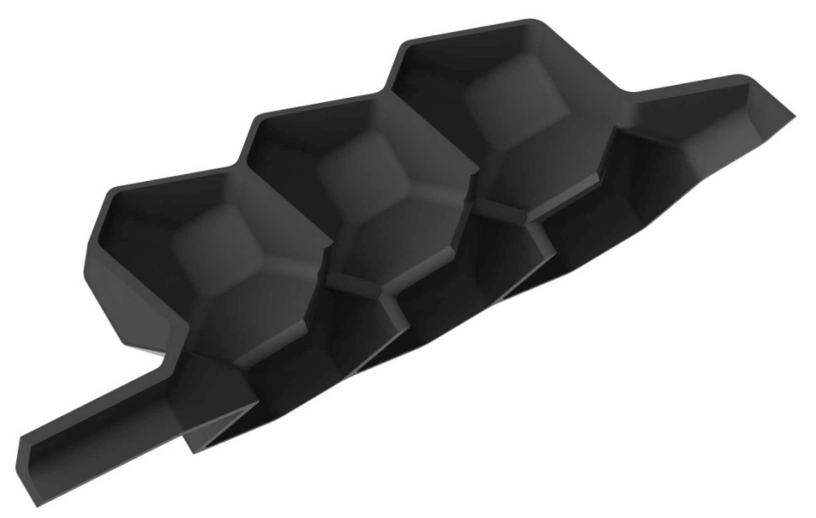

Figure 2. The one-sixth symmetry geometry.

\subsection{Governing equations and boundary conditions}

\subsubsection{Fluid domain}

Fluid flow is governed by the incompressible continuity and Navier-Stokes equations:

$$
\begin{aligned}
& \rho_{f} \frac{\partial v_{i}}{\partial x_{i}}=0 \\
& \rho_{f} \frac{\partial v_{i}}{\partial t}+\rho_{f} v_{i} \frac{\partial v_{i}}{\partial x_{j}}=-\frac{\partial p}{\partial x_{i}}+\mu \frac{\partial^{2} v_{i}}{\partial x_{j} \partial x_{j}},
\end{aligned}
$$

where $\rho_{f}=1.185 \mathrm{~kg} / \mathrm{m}^{3}$ is the fluid density, $\mu=$ $1.785 \mathrm{e}-5$ Pa.s is the fluid viscosity, $v_{i}$ is the velocity vector, $x_{i}$ is the position vector, $t$ is time, and $p$ is the fluid pressure.

Since an Eulerian system does not track the path of any fluid element, it cannot solve fluid freesurface problems directly. Therefore, the governing equations of fluid flow using an Arbitrary Lagrangian Eulerian (ALE) formulation are used by ADINA. In this method, the moving domain is recast at each time to a fixed configuration through ALE mapping, and the time derivative is computed on a reference configuration. The ALE equations are derived by substituting the relationship between the material time derivative and the reference configuration time derivative. In the discrete fluid flow problem, the change of the domain must be followed by a change in the discretization. The discretization of the new domain can be obtained through a re-meshing process or through a nodal relocation process. There exist several techniques to produce the nodal relocation. The simplest one is the Laplacian method which consists in solving the Laplace equation to update the node positions. ADINA also uses Laplace method but with a better algorithm to solve arbitrary moving meshes.

The boundary of the fluid domain is divided into the following regions for the assignment of boundary conditions: inlet, fixed walls, and FSI walls. There is no need to set the flow boundary condition at the inlet and it is allowed to be specified automatically during
FSI solution. In order to stabilize our simulation, we have set the inlet pressure to zero as the reference pressure. The FSI boundary condition is applied on the peripheral faces, except on the two lateral faces of the inlet channel, which are considered as fixed walls. The end face of our alveolar model is a closed end and is also an FSI boundary. Moreover, the symmetry condition is applied on the two symmetry planes of the model.

\subsubsection{Structural domain}

In contrast to the ALE formulation for the fluid equations, a Lagrangian coordinate system is implemented on the solid tissue domain in ADINA, and its deformation is governed by the momentum conservation equation given by Eq. (3):

$$
\rho_{s} \frac{\partial^{2} d_{i}^{s}}{\partial t^{2}}=\frac{\partial \sigma_{i j}^{s}}{\partial x_{j}}
$$

where $\rho_{s}=1050 \mathrm{~kg} / \mathrm{m}^{3}$ is the solid density, and $\sigma_{i j}^{s}$ and $d_{i}^{s}$, respectively, stand for the solid Cauchy stress tensor and solid displacement.

\section{Material models}

The wall material adopted in this work represents a tissue of average characteristics consistent with actual mechanical behavior of the alveolar wall tissue. As a first approximation, linear elastic material is used similar to previous works [14]. A Young's modulus of $8 \mathrm{kPa}$ and a Poisson's ratio of $v=0.42$ are used for the linear elastic model. The hyperelastic model includes a nonlinear relation between stress $(\sigma)$ and stretch of tissue $(\lambda)$ according to experimental stressstrain data [13] expressed by Eq. (4):

$$
\sigma=\lambda^{8}-\lambda^{6.4} \mathrm{kPa}
$$

We used a general Mooney-Rivlin hyperelastic material model. The strain energy function for the 9-parameter Mooney-Rivlin model of a hyperelastic material is expressed by nine constants. These constants are obtained by fitting the curve of Eq. (4).

The viscoelastic properties of the alveolar connective tissue were modeled using the quasi-linear viscoelastic (QLV) model of Fung [22]. In this theory, a relaxation function represents the force response to a step deformation.

The force response of a quasi-linear viscoelastic material at time $t$ is:

$$
F(t)=\int_{-\infty}^{t} G(t-\tau) \frac{\partial F^{e}(\delta(\tau))}{\partial \delta} \frac{\partial \delta(\tau)}{\partial t} d t,
$$

where $\partial F^{e} / \partial \delta$ is the instantaneous response and $\partial \delta / \partial \tau$ is the strain history of the material. This relation shows how the force response of a viscoelastic material to a constant deformation could vary with the time. 
The reduced relaxation function, for alveolar wall tissue is given by [23]:

$$
G(t)=0.4+0.6(t+1)^{-0.5} \text {. }
$$

The stress response of viscoelastic material is given by Eq. (7).

$$
\begin{aligned}
\sigma(t)= & \sigma_{0}[\varepsilon(t)]+\int_{0}^{t} \sigma_{0}[\varepsilon(t-\tau)] \\
& \frac{\partial G(\tau)}{\partial \tau} d \tau=\sigma_{0}[\varepsilon(t)]+H[\varepsilon(t), t],
\end{aligned}
$$

where $\sigma(t)$ is the dynamic stress response, $\sigma_{0}[\varepsilon(t)]$ is the instantaneous stress response to a strain $\varepsilon(t), t$ is the time, and $H[\varepsilon(t), t]$ is a history integral. $\sigma_{0}$ is defined by the nine-parameters Mooney-Rivlin material model and the effect of viscoelasticity is incorporated into it through Prony-Dirichlet series coefficients which have been obtained by curve fitting of the stress relaxation data.

We have chosen a Prony series expansion of the reduced relaxation function of the tissue which has the form:

$$
G(t)=\sum_{i=1}^{n} g_{i} \exp \left(\frac{-t}{\tau_{i}}\right)
$$

where $g_{i}$ is the $i$ th Prony series parameter, and $\tau_{i}$ is the corresponding Prony retardation time constants. We can find $g_{i}$ and $\tau_{i}$ by a nonlinear least squares optimization technique, such as the Levenberg-Marquardt algorithm [24]. A fourth-order Prony series, which is curve-fitted to the above reduced relaxation function, is found to have an excellent goodness of fit (residue fitness $<2 \mathrm{E}-6$ ). Table 1 lists the estimated Prony series parameters.

\section{Surface tension effect}

The inner surface of the alveoli is covered by a liquid lining which contains surfactant molecules. The role of these surface active agents is to lower the surface tension and facilitate the inflation and deflation of the alveoli. Many experiments have shown that the surface tension of the liquid lining including the surfactant has a dynamic behavior against the relative surface area. On the other hand, the expansion and compression behaviors are not similar and there is some hysteresis. Considering experimental work performed by $\mathrm{Lu}$ et al. (1998), one can see that at a surface ratio lower than $32 \%$, the expansion and compression curves coincide.

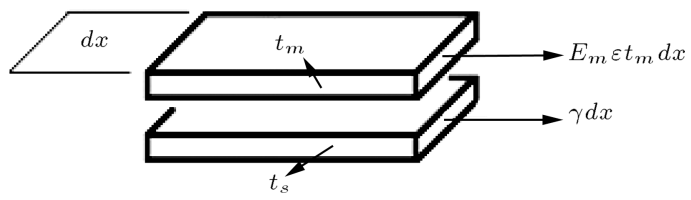

Figure 3. Including of surface tension of liquid layer in mechanical properties.

The result of Denny and Schroter's model [23] also confirmed that it is a good approximation to use similar curves for both inhalation and exhalation in surface ratios below $30 \%$.

So, in the normal breathing condition with low volume expansion, a similar relation can be used both in inhalation and exhalation. The relation between surface tension $(\gamma)$ and relative surface area $\left(\frac{S}{S_{0}}\right)$ is achieved by linear curve fitting of their results as follows:

$$
\gamma=80.5\left(\frac{S}{S_{0}}-1\right)+2\left[\frac{m N}{M}\right]
$$

Let us consider an element of the alveolar tissue with an elastic modulus of $E_{m}$, thickness of $t_{m}$, and width of $d x$ stretched by strain of $\varepsilon$. The resulting force is given by $E_{m} \varepsilon t_{m} d x$. Now, imagine the liquid lining with a surface tension, $\gamma$, attached to the tissue as shown in Figure 3. Then, the surface tension force of this liquid layer is $\gamma d x$.

It is favorable to replace these two layers with an equivalent tissue which has the same stiffness. For this purpose, the effective force should be equal to the sum of the forces acting on the two layers.

$$
F=E_{m} \varepsilon t_{m} d x+\gamma d x=\sigma_{\text {total }} t_{m} d x .
$$

So, the elastic stress of the equivalent tissue is:

$$
\sigma_{\text {total }}=\sigma_{m}(\varepsilon)+\frac{\gamma}{t_{m}} .
$$

If the surface tension $\gamma$ is expressed as a function of $\varepsilon$, then a new stress-strain relation for the equivalent tissue can be obtained.

As previously reported, surface tension is related to relative surface area. So, using the following generally accepted relationship between stretch $\lambda$ and relative surface area, $(1+\varepsilon)^{2}$ can be substituted for $\frac{S}{S_{0}}$ and the new stress-strain behavior for the equivalent alveolar tissue including surface tension is achieved. It should be noted that $\sigma_{m}(\varepsilon)$ is stress-strain behavior of the tissue material model:

Table 1. Prony series coefficients.

\begin{tabular}{cccccccc}
\hline $\boldsymbol{g}_{\mathbf{1}}$ & $\boldsymbol{\tau}_{\mathbf{1}}$ & $\boldsymbol{g}_{\mathbf{2}}$ & $\boldsymbol{\tau}_{\mathbf{2}}$ & $\boldsymbol{g}_{\mathbf{3}}$ & $\boldsymbol{\tau}_{\mathbf{3}}$ & $\boldsymbol{g}_{\mathbf{4}}$ & $\boldsymbol{\tau}_{\mathbf{4}}$ \\
\hline 0.0878 & 0.5331 & 0.2058 & 2.0304 & 0.0642 & 3.9905 & 0.6413 & 100.0002 \\
\hline
\end{tabular}




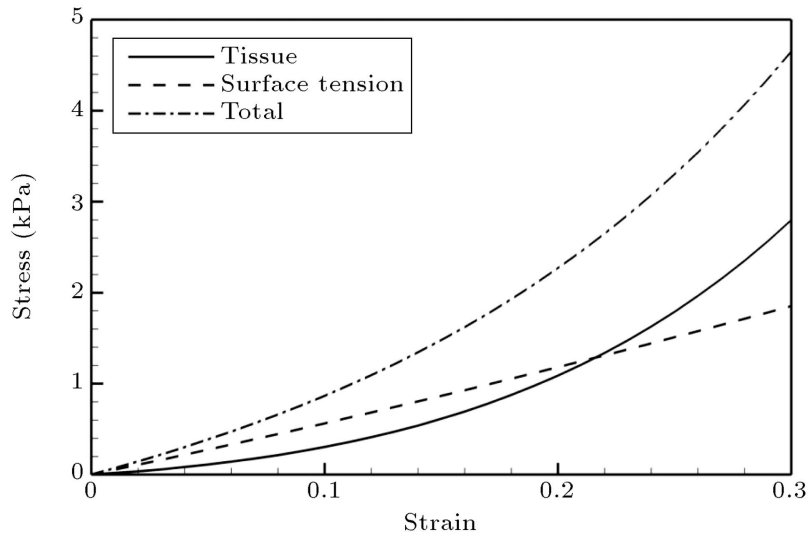

Figure 4. Stress-strain curve of the equivalent tissue including surface tension.

$$
\begin{aligned}
& \lambda=L / L 0=\left(S / S_{0}\right)^{1 / 2}=\left(V / V_{0}\right)^{1 / 3}, \\
& \frac{S}{S_{0}}=(1+\varepsilon)^{2}, \\
& \sigma_{\text {total }}(\varepsilon)=\sigma_{m}(\varepsilon)+\frac{80.5\left[(1+\varepsilon)^{2}-1\right]}{t_{m}} .
\end{aligned}
$$

The constant term of the surface tension (Eq. (9)), which is the initial surface tension of the liquid layer at the start of the inhalation, is omitted because, including this term in a stress-strain relation would unbalance the system at the start of inhalation. Physiologically, at the start of the inhalation, there is an equal force in the reverse direction originated by the pleural fluid suction which counterbalances it and results in an equilibrium initial state.

The stress-strain relation of equivalent tissue including surface tension is plotted in Figure 4. It can be seen that including surface tension in the material model cause the tissue to become stiffer. For curve fitting parameters for Mooney-Rivlin model with surface tension effect refer to Table 2 .

Actually the deformation of the alveolated system is produced by the action of both the air and the other tensions coming from physiology. The difference between air pressure and pleural pressure (transpulmonary pressure or recoil pressure) balances with the sum of the alveolar tissue tension and surface tension. Also in the work of Wilson and Bachofen [25], it has been declared that the tissue tension and surface tension are working together and balances the recoil pressure. Recoil pressure is applied as our boundary condition which causes wall deformation. Consequently, wall deformation produces the tissue and surface tensions which balance with the recoil pressure. We do not model the surface liquid layer separately and, instead, we include the effect of the surface tension of the surface lining liquid with surfactant into our material properties. Hence, the surface tension acts as a material stiffening agent which withstands the alveolar inflation and facilitates alveolar deflation.

\section{Boundary conditions}

To find the proper boundary condition at the outer surface of the alveolar tissue, we have focused on the mechanics of normal respiration. When the lungs are at rest, the inward elastic recoil of the alveolar walls balances the outward elastic recoil of the chest wall. The two opposing forces of chest elastic recoil and lung elastic recoil cause the intrapleural pressure to be negative relative to atmospheric pressure. Transpulmonary pressure is defined as the difference between alveolar pressure and pleural pressure Ptp $=(P a l v-P p l)$. According to physiology handbooks [20] it increases from 500 to $700 \mathrm{~Pa}$ during inhalation and, then, decreases during exhalation. The transpulmonary pressure, at the resting volume, represents the natural tendency of the alveoli to collapse or the chest wall to move outward. So, the transpulmonary pressure gradient is responsible for maintaining alveolar expansion.

We assume that our simulation starts from the resting volume. So, the transpulmonary pressure gradient varies from 0 to $200 \mathrm{~Pa}$ which is similar to the assumption of [14]. It is more physiologically accurate to impose transpulmonary pressure instead of alveolus inlet flow rate as the boundary condition. For this reason, we have sought a pressure profile which has the best agreement with experimental observations. Assuming the same transient flow rate behavior throughout each airway branch and acinus of the lung, the experimental curve of tidal breathing flow rate [8], obtained from spirometry, could be assumed to approximate the alveolar flow rate (Figure 5). In order to remove the experimental fluctuations, we have used two-piece polynomial curves fitting to the spirometry data. Considering that the pressure-volume relation is linear for small tidal volume excursions, the shape of the transpulmonary pressure could be approximated by integrating the experimental curve of tidal breathing flow rate [8] and scaling it in such a way that its peak reaches $200 \mathrm{~Pa}$ and the breathing period fits $4 \mathrm{~s}$.

Although this profile does not accurately represent the transpulmonary pressure, it is more precise than the ideal sinusoidal profile employed by Dailey and Ghadiali [14]. As is known, the pleural pressure

Table 2. Mooney-Rivlin parameters for tissue material by including surface tension effect.

\begin{tabular}{cccccccccc}
\hline $\begin{array}{c}\text { Mooney-Rivlin parameters with } \\
\text { surface tension effect }\end{array}$ & C1 & C2 & C3 & C4 & C5 & C6 & C7 & C8 & C9 \\
\cline { 2 - 9 } & -1.55 & 2.90 & -30.86 & 4.54 & 39.71 & 22.95 & -51.76 & 22.60 & 46.99 \\
\hline
\end{tabular}




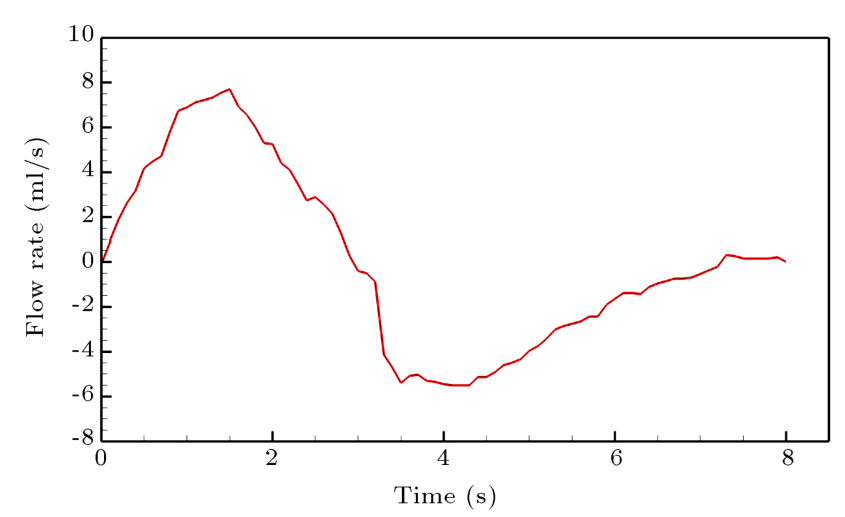

Figure 5. Experimental unsteady flow rate curve used to simulate breathing conditions in healthy alveolar sac model [8].

acts on the visceral pleura $(25-83 \mu \mathrm{m})$ which covers the lung parenchyma including the whole of the alveoli connecting to each other. So, in the simulation of an isolated alveolus, the magnitude of the imposed pressure should be considerably decreased to consider the effect of the tissue surrounding the alveolar sac. Hence, we have scaled the pressure profile in order to achieve a physiological volume expansion. Choosing a coefficient of 0.25 , we obtained a volume expansion of the viscoelastic model which is confirmed by the results of Harding Jr. and Robinson [9] and Tsuda et al. [1]. The accepted scaled pressure profile for the boundary condition is shown in Figure 6.

The fluid and solid domains are coupled by satisfying the following boundary conditions at the interface between the two domains: (i) Displacements of the fluid and solid domain FSI boundaries must be equal; and (ii) Tractions at FSI boundaries must be balanced. These conditions are given by Eqs. (15) and (16).

$$
\begin{aligned}
& d_{i}^{f}=d_{i}^{s}, \\
& n_{j} \sigma_{i j}^{f}=n_{j} \sigma_{i j}^{s},
\end{aligned}
$$

where:

$$
\sigma_{i j}^{f}=-p \delta_{i j}+\mu\left(\frac{\partial v_{i}}{\partial x_{j}}+\frac{\partial v_{j}}{\partial x_{j}}\right),
$$

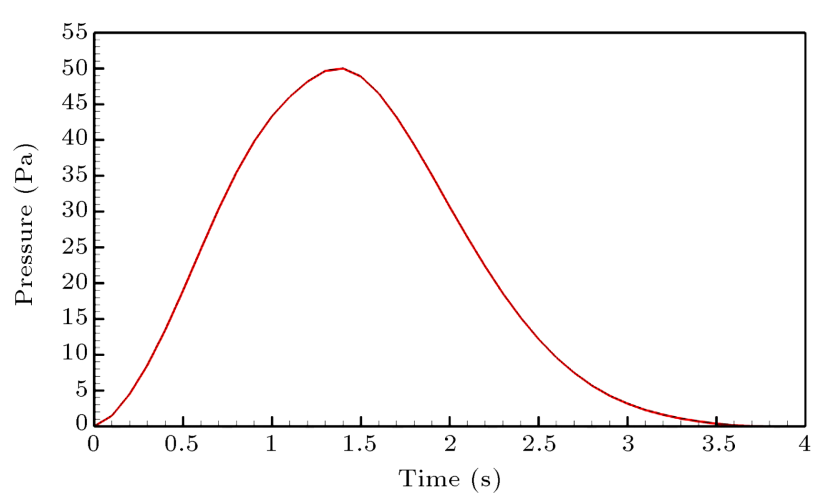

Figure 6. Pressure profile boundary condition. where $d_{i}^{f}$ and $d_{i}^{s}$ are the fluid and solid nodal displacements, $n_{j}$ is the interface normal vector, $\delta_{i j}$ is the Kronecker delta function, and $\sigma_{i j}^{f}$ and $\sigma_{i j}^{s}$ are the fluid and solid stress tensors.

On the two symmetry planes, the symmetry boundary condition is applied on both fluid and solid domains. So, the solid displacement and fluid velocity normal to these planes are set to zero.

\subsection{Fluid-structure interaction implementation}

The software ADINA 8.8 was utilized for our timedependent simulation of FSI between the alveolar wall and the lumen. The ADINA program is a generalpurpose finite element/finite volume software for the analysis of structures, fluids and FSI problems [26]. Structures are modeled using finite element procedures. An Arbitrary Lagrangian-Eulerian (ALE) formulation is employed when there is movement of the fluid boundaries. The Navier-Stokes equations of incompressible flows are solved using finite element procedures.

In order to coupling between the fluid and structural models, there are two methods of direct and iterative in ADINA [27]. The difference between these methods is that in the direct method the fluid and solid equations are solved simultaneously, while in the iterative method they are solved successively. The fluid nodal positions on the fluid-structure interfaces are determined by the kinematic conditions. The displacements of the other fluid nodes are determined automatically by the program to preserve the initial mesh quality. The governing equations of fluid flow in their ALE formulations are then solved. According to the dynamic conditions, on the other hand, the fluid traction is integrated into fluid force along fluidstructure interfaces and exerted onto the structure node. The solid and fluid tractions are compared and in the case of any difference, iterations are continued with the updated traction and displacement information at the fluid-solid interface. The routine applies calculated forces onto the structural model, evaluates incremental structural displacements, applies these incremental structural displacements onto the fluid model, and continues to iterate. Finally, when interface boundary conditions are satisfied within an acceptable tolerance, the information is stored and another time step is started.

The direct solution procedure in ADINA solves the governing equations for the structure and the fluid as the partitioned procedure, but obtains the solution by solving these equations simultaneously using one coefficient matrix. This matrix contains the same structural and fluid matrices as in the partitioned procedure plus a Jacobian matrix corresponding to the interface conditions, obtained by imposing directly the conditions by Eqs. (16) and (17). 


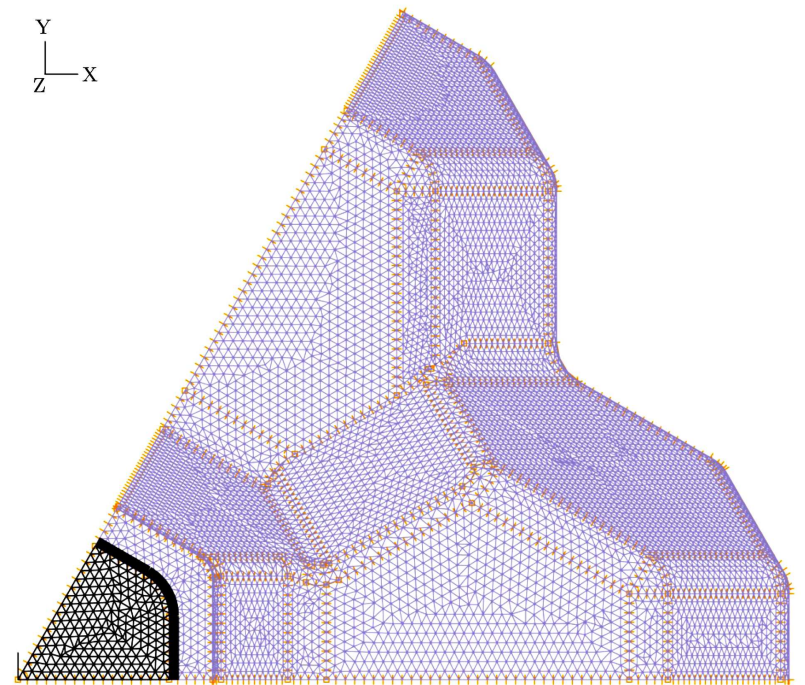

Figure 7. Generated tetrahedral mesh.

Since usually a much finer fluid mesh than structural mesh is needed, the conditions of compatibility require the displacement and stress interpolations of the structural elements.

We have used the direct method for two-way coupling between the fluid and solid domain which is more applicable to solving large deformations with soft materials. These details can be found on ADINA guide [26] and other FSI references [28,29].

In this work, we make use of tetrahedral elements for solid and fluid to discretize the domains in ADINA. The model is composed of 533,000 elements for the solid and 1,273,000 elements for the fluid domains (Figure 7). Mesh sensitivity analyses were conducted with three different mesh sizes ranging from 240,000 to $3,550,000$ fluid elements and 88,000 to $1,000,000$ solid elements with average mesh size of $0.008,0.004$, and $0.002 \mathrm{~mm}$. Independence in mesh size was obtained for the resulting alveolus volume and showed less than $1 \%$ relative error for the medium (0.004) and fine (0.002) meshes (Figure 8). CPU time was about 4-5 days with an Intel $\mathrm{i} 5-2500 \mathrm{k}(4 \times 3.3 \mathrm{GHz})$ system with 8 GB RAM.

\section{Results and discussions}

We have simulated normal breathing for three different types of alveolar wall materials. In each case, we computed the time variation of the alveolus volume. Differentiation of this curve with time gives us the inlet flow profile as shown in Figure 9. In the case of a viscoelastic wall, four successive cycles have been simulated to reach steady state conditions in which the relative change of maximum flux falls below $2 \%$.

A Young's elastic modulus of $8 \mathrm{kPa}$ gives a flux profile with a maximum of $0.5 \mathrm{~s}$ and amplitude of $4 \mathrm{ml} / \mathrm{s}$. The maximum of the flow profile with

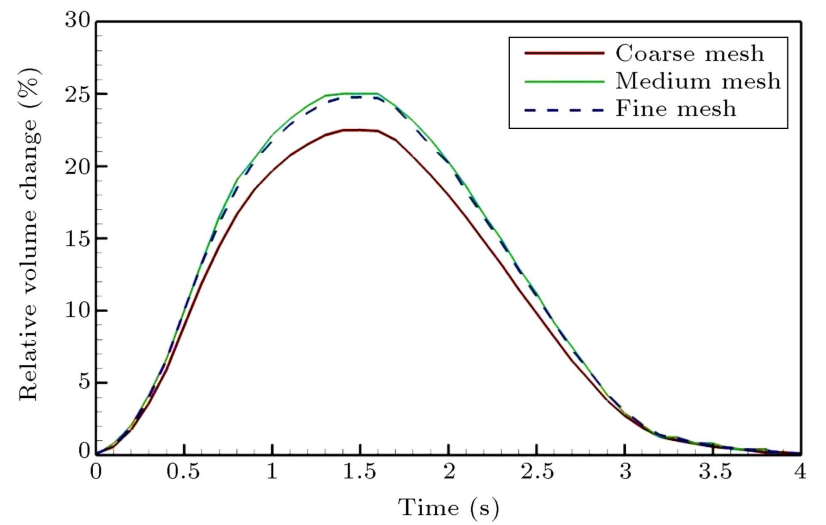

Figure 8. Mesh sensitivity analysis by comparing alveolus volume.

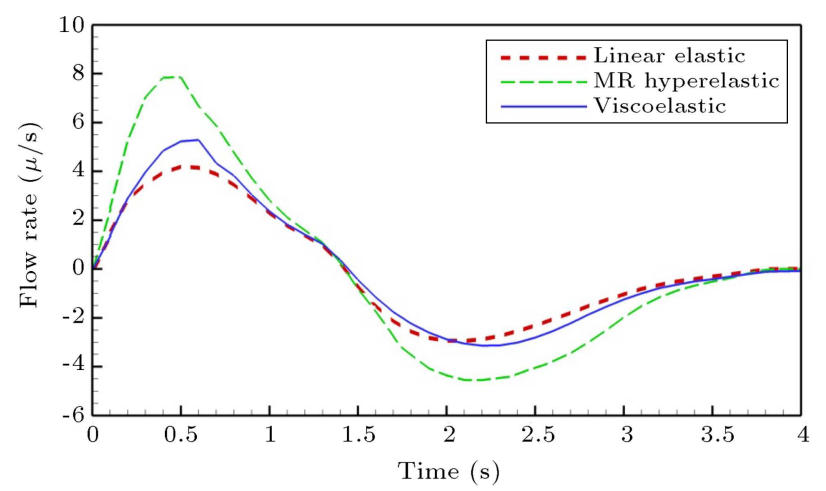

Figure 9. Resulting air fluxes using different material models.

hyperelastic material in the Mooney Rivlin model is $7.8 \mathrm{ml} / \mathrm{s}$ at $0.5 \mathrm{~s}$ while in the viscoelastic model it is $5 \mathrm{ml} / \mathrm{s}$ at $0.6 \mathrm{~s}$. This means that adding the viscoelastic effect will shift the maximum flow to the right side relative to the elastic models, and also decrease the respiratory flow rate. This phase shift is also observed in the FSI simulation of the viscoelastic thin wall model for stenotic arteries performed by Chen [30]. The magnitude of the phase lag is about 10 degree which is validated by Miki et al. [31].

In order to acquire the compliance diagram of our model, the alveolus volume at each time step is plotted against the pleural pressure. An illustration of the compliance of the model is given in Figure 10 . Typically, the Pressure-Volume (PV) curve in Acute Respiratory Distress Syndrome (ARDS) patients from Functional Residual Capacity (FRC) to Total Lung Capacity (TLC) has a sigmoidal shape. This is similar to the shape of the curve from Residual Volume (RV) to TLC in healthy humans. In this study, inflation and deflation of a healthy alveolus during normal breathing, from FRC to FRC+TV (Tidal Volume), is simulated. So we do not expected to see the lower and upper inflection points of a patient PV curve during mechanical ventilation. It should be emphasized that it cannot be expected that this simple model resembles 


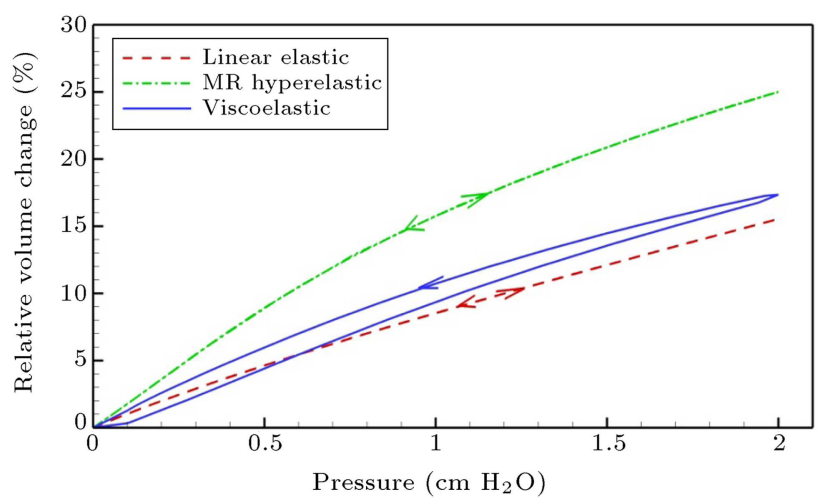

Figure 10. Compliance diagram for different material models.

the overall PV relation of the lung. It is a useful insight into air flow within terminal parts of the lung.

When plotting a single breath on a PV curve, based on the properties of elastance, we would have the same volume for the same pressure (same points on PV curve) on inspiration as on expiration. However, a normal compliance diagram shows that during deflation, the lung volume at any given pressure is greater than during inflation. This behavior is called hysteresis. As shown in Figure 10, the compliance curves for the linear elastic and hyperelastic model have linear and nonlinear relations, respectively, but do not show any hysteresis behavior. In contrast, the viscoelastic model is in reasonable qualitative agreement with published experimental curves [32] and also shows the hysteresis behavior of the alveolar tissue. It should be mentioned that all three model parameters come from curve fitting and are not arbitrary. The same stressstrain relationship has been used to find the material parameters in all models and the only difference is about adding relaxation function in viscoelastic model. So, our finding about hysteresis behavior can only be related to viscoelastic model.

So, the viscoelastic model including surface tension effect was utilized to study the characteristics of flow pattern in the alveolus and also the distributions of stresses and deformations within the alveolar wall.

The velocity contours and streamlines at instant of maximum inhalation $(t=0.6 \mathrm{~s})$ are shown in Figure 11 for viscoelastic model. The maximum velocity

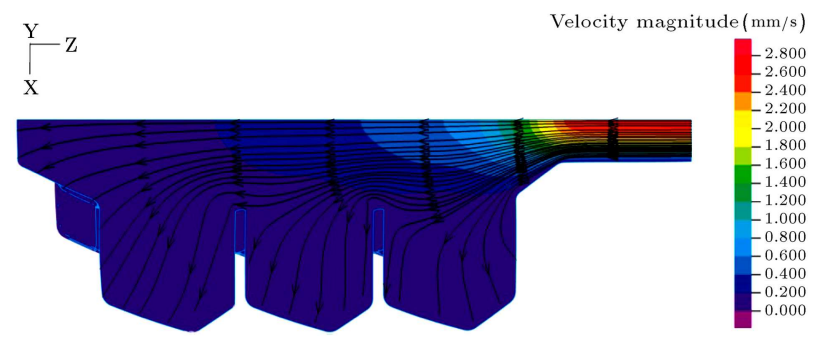

Figure 11. Air velocity contours and streamlines at $t=0.6 \mathrm{~s}$.

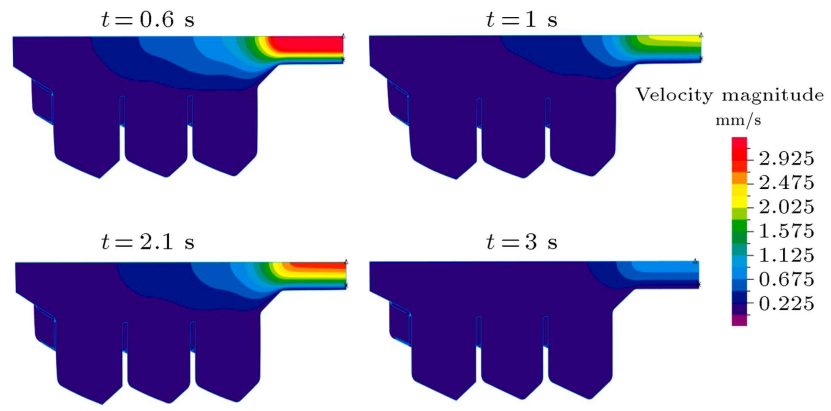

Figure 12. Velocity contours at certain times: (a) $t=0.6$ $\mathrm{s}$; (b) $t=1 \mathrm{~s}$; (c) $t=2.1 \mathrm{~s}$; and (d) $t=3 \mathrm{~s}$.

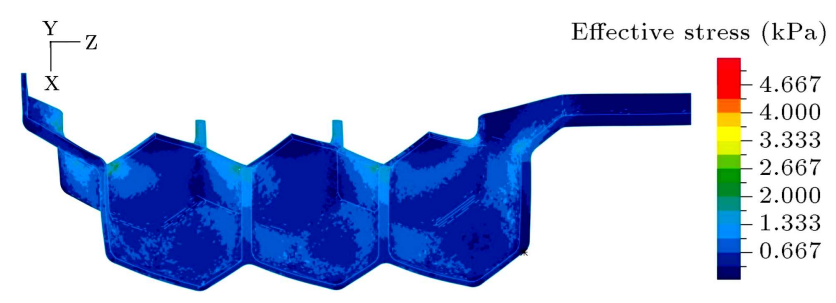

Figure 13. Effective stress contours at $t=1.4 \mathrm{~s}$.

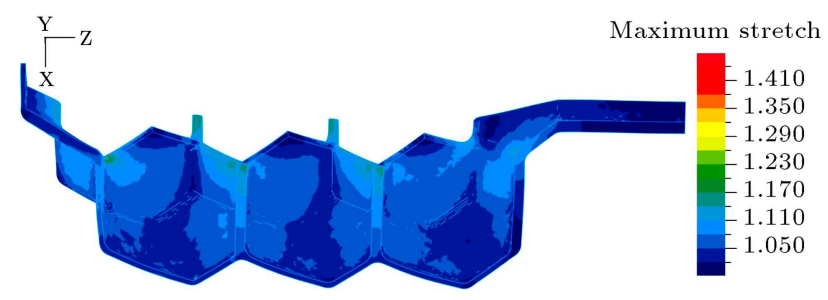

Figure 14. Maximum stretch contours at $t=1.4 \mathrm{~s}$.

magnitude at this time is $2.8 \mathrm{~mm} / \mathrm{s}$ and streamlines show a laminar radial flow with no circulation because of the very low velocities at the end alveolus. This observation is in good agreement with reported radial airflow in the previous literature $[7-9,11]$. It shows that for acinar flow rates similar to those examined in this study, the flow is reversible and no convective mixing is present in the terminal alveolus.

The velocity contours at different times for viscoelastic model are shown in Figure 12. The maximum air velocity entering and leaving the alveolus occurs at $t=0.6 \mathrm{~s}$ and $t=2.1 \mathrm{~s}$, respectively.

The distribution of effective stress and the maximum stretch ratio in alveolar tissue, at the time in which the applied pressure is at a maximum $(t=$ $1.4 \mathrm{~s})$ for viscoelastic model, are depicted in Figures 13 and 14. According to these figures the local values are much higher than the global values. These uneven stress and strain distributions could be expected due to thinner walls near corners which has less tissue to resist the deformation. Thin regions become overstretched while the other regions are not involved. The change of maximum local stretch during one breath for viscoelastic model is also plotted in Figure 15 . As it can be seen, the maximum local strain is $20 \%$ and it 


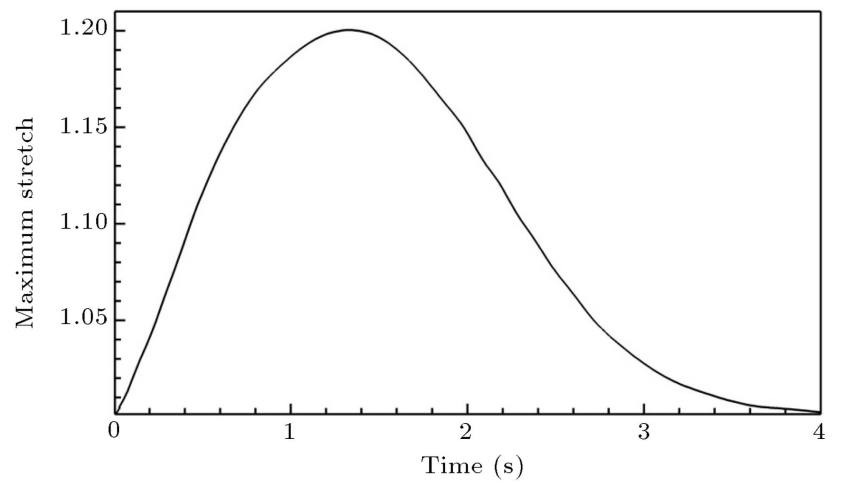

Figure 15. Variation of maximum local stretch during breathing time.

occurs at the time $t=1.4 \mathrm{~s}$. The maximum global stress and strain in the time of $t=1.4 \mathrm{~s}$ are $1 \mathrm{kPa}$ and 0.05 , respectively, while in hotspots the maximum stress and strains reach to $2 \mathrm{kPa}$ and 0.2 . Rausch et al. [33], utilizing synchrotron-based X-ray tomographic microscopy, also found a maximum local strain of 0.2 .

Experimental studies have shown that high transpulmonary pressures, resulting from ventilation, may damage the capillary walls, thereby leading to edema. So, finding the stress and strain distribution within the alveolar region would be an important step in understanding this phenomenon. It is generally estimated that the alveolar cells are exposed to 1$5 \%$ strain during normal breathing, and larger strains in mechanical ventilation could cause inflammatory reactions on alveolar type II or alveolar epithelial cells. Chandel and Sznajder [34] reported that high cyclic stretch frequencies up to 60 cycles/min are another reason for edema. Our strain results are in accordance with these physiological findings. The larger strains existing in the model corners could not cause edema considering the normal breathing frequency of $15 \mathrm{cy-}$ cles/min but the increase in local strain predicts that edema could potentially initiate at a lower level than previously thought.

In the most of lung diseases, the mechanical properties of lung tissue has been changed. Usually, the tissue becomes stiffer and lung compliance decreases. Our model could also be used to predict the alveolar mechanics under most of the pathological conditions by changing the material model used for the alveolar wall.

\section{Conclusion}

In this study, using FSI analysis on a 3D model of the alveolar sac, a new model for passive breathing is utilized.

The pressure boundary condition applied in this simulation is compatible with the experimental respiratory flow rate. In contrast to the literature in which the air flow is predefined, in this model the applied external pressure causes the air to flow in and out of the alveolus.

This study confirmed that the air flow pattern in the terminal alveolus is radial with no circulation. Moreover, this FSI model could provide actual air flow patterns for further findings such as particle and gas transport in healthy or patient lungs.

This model has the advantage that we will be able to find local alveolar stress and strain fields by incorporating the mechanical properties of alveolar tissue. Since, the lung parenchyma has nonlinear viscoelastic properties, we investigated the effect of viscoelastic behavior of alveolar tissue on the profile of the flow rate. Wall viscoelasticity causes a phase shift in the flow profile relative to other elastic models and shows the hysteresis behavior of the compliance diagram. Maximum distributions of effective stress and stretch of $2 \mathrm{kPa}$ and 1.2 , respectively, are reported. The simulation results show that there are certain hotspots in the alveolus which are at risk for edema which are located in thinnest regions.

In conclusion, including the mechanical properties of the alveolar wall in FSI simulation gives us a better description of the alveolar mechanics. Based on the results, the nonuniform expansion of the alveolus suggests that segments that distend the most might be preferred alveolar locations for injury. The potential applications of the present work to the clinical situation of mechanically ventilated patients are to investigate how ventilation strategies affect the deformations at the alveolar level and could cause overstretching.

\section{Nomenclature}

$\begin{array}{ll}\text { FRC } & \text { Functional Residual Capacity } \\ \text { FSI } & \text { Fluid-Structure Interaction } \\ \text { PV } & \text { Pressure-Volume } \\ \text { R } V & \text { Residual Volume } \\ \text { TLC } & \text { Total Lung Capacity } \\ \text { TV } & \text { Tidal Volume } \\ d_{f, s} d_{f, s} & \text { Displacement vector ( } f \text { denotes fluid, } s \\ & \text { denotes solid) } \\ E & \text { Modulus of elasticity } \\ G & \text { Reduced relaxation function } \\ n_{i} & \text { Normal vector } \\ P & \text { Fluid pressure } \\ \text { Palv } & \text { Alveolar pressure } \\ P p l & \text { Pleural pressure } \\ \text { Ptp } & \text { Transpulmonary pressure } \\ v_{i} & \text { Velocity vector }\end{array}$

\section{Greek symbols}

$\delta_{i j} \quad$ Kronecker delta 
$\varepsilon$

$\lambda$

$\mu$

$\sigma_{0}$

$\sigma_{i j}^{f, s}$

$\rho_{f, s}$

Strain rate

Stretch ratio

Dynamic viscosity of the fluid

Instantaneous stress response

Cauchy stresses tensor ( $f$ denotes fluid, $s$ denotes solid)

Material density ( $f$ denotes fluid, $s$ denotes solid)

\section{References}

1. Tsuda, A., Henry, F.S. and Butler, J.P.,"Chaotic mixing of alveolated duct flow in rhythmically expanding pulmonary acinus", J. of Appl. Physiol., 79, pp. 10551063 (1995).

2. Henry, F.S., Butler, J.P and Tsuda, A. "Kinematically irreversible acinar flow: a departure from classical dispersive aerosol transport theories", J. of Appl. Physiol., 92, pp. 835-845 (2002).

3. Darquenne, C. and Paiva, M. "Two- and threedimensional simulations of aerosol transport and deposition in alveolar zone of the human lung", $J$. of Appl. Physiol., 80, pp. 1401-1414 (1996).

4. Harrington, L., Kim Prisk, G. and Darquenne, C., "Importance of the bifurcation zone and branch orientation in simulated aerosol deposition in the alveolar zone of the human lung", J. of Aerosol Sci., 37(1), pp. 37-62 (2006).

5. Haber, S., Butler, J.P., Brenner, H., Emanuel, I. and Tsuda, A., "Shear flow over a self-similar expanding pulmonary alveolus during rhythmical breathing", $J$. of Fluid Mech., 405, pp. 243-268 (2000).

6. Haber, S., Yitzhak, D. and Tsuda, A., "Gravitational deposition in a rhythmically expanding and contracting alveolus", J. of Appl. Physiol., 95, pp. 657-671 (2003)

7. Sznitman, J., Heimsch, F., Heimsch, T. Rusch, D. and Rösgen, T. "Three-dimensional convective alveolar flow induced by rhythmic breathing motion of the pulmonary acinus", J. of Biomech. Eng., 129, pp. 658665 (2007).

8. Oakes, J.M., Day. S., Weinstein, S.J. and Robinson, R.J. "Flow field analysis in expanding healthy and emphysematous alveolar models using particle image velocimetry", ASME J. of Biomech. Eng., 132(2), 021008 (2010).

9. Harding Jr., E.M. and Robinson, R.J. "Flow in a terminal alveolar sac model with expanding walls using computational fluid dynamics", J. of Inhalation Toxicology, 22(8), pp. 669-678 (2010).

10. Darquenne, C., L. Harrington, and Prisk, G.K. "Alveolar duct expansion greatly enhances aerosol deposition: a three-dimensional computational fluid dynamics study", Philosophical Transactions of The Royal Society, 367, pp. 2333-2346 (2009).
11. Berg, E.J., Weisman, J.L., Oldham, M.J. and Robinson, R.J. "Flow field analysis in a compliant acinus replica model using particle image velocimetry (PIV)", J. Biomech., 43(6), pp. 1039-1047 (2010).

12. Li, Z. and Kleinstreuer, C. "Airflow analysis in the alveolar region using the lattice-Boltzmann method", Med. Biol. Eng. Comput., 49(4), pp. 441-451 (2011).

13. Gefen, A., D. Elad, and Shiner, R.J. "Analysis of stress distribution in the alveolar septa of normal and simulated emphysematic", J. of Biomech., 32, pp. 891897 (1999).

14. Dailey, H.L. and Ghadiali, S.N. "Fluid-structure analysis of microparticle transport in deformable pulmonary alveoli", J. of Aerosol Sci., 38(3), pp. 269-288 (2007).

15. Schittny, J.C., Mund, S.I. and Stampanoni, M. "Evidence and structural mechanism for late lung alveolarization", Am. J. Physiol.-Lung Cell Mol. Physiol., 294, pp. 246-254 (2008).

16. Hwang, J., Kim, M., Kim, S. and Lee, J. "Quantifying morphological parameters of the terminal branching units in a mouse lung by phase contrast synchrotron radiation computed tomography", PLoS ONE, 8(5), pp. e63552 (2013).

17. Vasilescu, D.M., Gao, Z., Saha, P.K., Yin, L., Wang, G., Haefeli-Bleuer, B., Ochus, M., Weibel, E.R. and Hoffman, E.A. "Assessment of morphometry of pulmonary acini in mouse lungs by nondestructive imaging using multiscale microcomputed tomography", Proceedings of the National Academy of Sciences, 109(42), pp. 17105-10 (2012).

18. Kumar, H., Vasilescu, D.M., Yin, Y., Hoffman, E.A. Tawhai, M.H. and Lin, C.-L. "Multiscale imaging and registration-driven model for pulmonary acinar mechanics in the mouse", J. of Appl. Physiol., 114(8), pp. 971-8 (2013).

19. Sera, T., Yokota, H., Tanaka, G., Uesugi, K., Yagi, N. and Schroter, R.C. "Murine pulmonary acinar mechanics during quasi-static inflation using synchrotron refraction-enhanced computed tomography", J. of Appl. Physiol., 115, pp. 218-228 (2013).

20. Levitzky, M.G. Pulmonary Physiology, McGraw-Hill, 7th Edn. (2007).

21. Kumar, H., Tawhai, M.H., Hoffman, E.A. and Lin, C.L. "The effects of geometry on airflow in the acinar region of the human lung", J. of Biomech., 42(11), pp. 1635-1642 (2009).

22. Fung, Y.C., Biomechanics: Mechanical Properties of Living Tissues, Springer Verlag (1993).

23. Denny, E. and Schroter, R.C. "Viscoelastic behavior of a lung alveolar duct model", J. of Biomechanical Engineering, 122(2), pp. 143-151 (2000).

24. Press, W.H., Teukolsky, S.A., Vetterling, W.T. and Flannery, B.P. Numerical Recipes in C: The Art of Scientific Computing, 2nd Edn., Cambridge University Press (1992) 
25. Wilson, T.A. and Bachofen, H. "A model for mechanical structure of the alveolar duct", J. Appl. Physiol., 52(4), pp. 1064-1070 (1982).

26. ADINA R\&D, Inc., ADINA 8.8 "Automatic dynamic incremental nonlinear analysis theory and modeling guide" I, II, III (2011).

27. Zhang, H. and Bathe, K.J. "Direct and iterative computing of fluid flows fully coupled with structures", First MIT Conf. on Comput. Fluid and Solid Mech. (2001).

28. Garelli, L. "Fluid Structure Interaction using an Arbitrary Lagrangian Eulerian Formulation", PhD thesis, Faculted de Ingenieíra y Ciencias Hídricas (2011).

29. Hou, G., Wang, J. and Layton, A. "Numerical methods for fluid-structure interaction: a review", Commun. Comput. Phys., 12(3), pp. 337-377 (2012).

30. Chen, X. 'A Nonlinear Viscoelastic Mooney-Rivlin Thin Wall Model for Unsteady Flow in Stenotic Arteries, in Applied Mathematics Department, Worcester Polytechnic Institute (2003).

31. Miki, H., Bulter, J.P., Rogers, R.A. and Lehr, J.L. "Geometric hysteresis in pulmonary surface-to-volume ratio during tidal breathing", J. of Appl. Physiol., 75, pp. 1630-1636 (1993).

32. Lai-Fook, S.J., Wilson, T.A., Hyatt, R.E. and Rodarte,
J.R. "Elastic constants of inflatedlobes of dog lungs", J. Appl. Physiol., 40, pp. 508-513 (1976).

33. Rausch, S.M.K., Haberthur, D. and Stampanoni, M. "Local strain distribution in real three-dimensional alveolar geometries", Ann. Biomed. Eng., 39(11), pp. 2835-2843 (2011).

34. Chandel, N.S. and Sznajder, J.I. "Stretching the lung and programmed cell death", Am. J. Physiol., 279(6), pp. 1003-1004 (2000).

\section{Biographies}

Mojdeh Monjezi is a PhD candidate at the Center of Excellence in Energy Conversion, School of Mechanical Engineering, Sharif University of Technology (SUT). She received her $\mathrm{BSc}$ and $\mathrm{MSc}$ degrees at Isfahan University of Technolog. Her research interests are biofluid simulation, modeling of particle transport and respiratory systems and aerosol dynamics.

Mohammad Said Saidi is the Professor of Mechanical Engineering at Sharif University of Technology. His research interests are modeling and numerical analysis of transport and deposition of aerosol particles, modeling and numerical analysis of biofluids, modeling and numerical analysis of thermal-hydraulics of porous media and microchannels. 